correlated with vertebral destruction, for this reason, patients with this finding should be more carefully follow-up.

Disclosure of Interest: None declared

DOI: 10.1136/annrheumdis-2018-eular.7272

\section{SAT0401 RISK OF HOSPITAL ADMISSION DUE TO SEVERE INFECTION IN PATIENTS UNDER TREATMENT WITH ANTI-TNF DRUGS: DATA FROM A LOCAL REGISTRY}

J. Rosas $^{1}$, M. Pucciarelli ${ }^{2}$, J.M. Senabre-Gallego ${ }^{1}$, J.A. García-Gómez ${ }^{3}, J$. Ena ${ }^{2}$, G. Santos-Soler ${ }^{1}$, X. Barber ${ }^{3}$, E. Salas ${ }^{1}$, A. Pons-Bas ${ }^{1}$, C. Cano ${ }^{1}$, E. Ivars ${ }^{1}$, M. Lorente ${ }^{1}$ on behalf of AIRE-MB Group. ${ }^{1}$ Rheumatology Department, ${ }^{2}$ Internal Medicine Department, Hospital Marina Baixa, Villajoyosa (Alicante); ${ }^{3} \mathrm{COI}$, Miguel Hernández University, Elche, Spain

Objectives: To Know characteristics of patients treated with anti-TNF, who suffered infections that forced hospital admission.

Methods: Prospective observational study in patients treated with anti-TNF, during $1 / 1 / 2000$ to $12 / 31 / 2017$, followed up in the Rheumatology Section. General data of patients (age, gender), of disease (diagnosis and time of evolution, type of anti-TNF, time in anti-TNF, concomitant treatment with DMARD), regarding the presence of severe infection, defined as infection that required hospital admission (time in anti-TNF to infection, location of infection, days of admission, mantoux/ IGRAS and vaccinations prior to the start anti-TNF treatment) was collected. The admission decision was made by Emergency Department of centre or Rheumatology.

Results: Of 442 patients with anti-TNF, $44(9.6 \%)$ patients had at least one hospital admission due to severe infection. $59 \%$ were women, with mean age $64 \pm 16.72$ years, ${ }^{22-88} 21.25 \pm 4.02$ years of disease evolution. A mantoux/IGRAS was performed prior anti-TNF. Diagnosis was: rheumatoid arthritis (RA): $25(57 \%)$, ankylosing spondylitis (AS): $12(27 \%)$, psoriatic arthritis (PSA): $5(11 \%)$ and juvenile idiopathic arthritis (JIA): $2(5 \%)$. The mean time of treatment with anti-TNF is 5.6 \pm 4.5 years. Adalimumab received $24(55 \%)$ patient, infliximab $8(18 \%)$ patients, etanercept $6(14 \%)$ patients, golimumab $5(11 \%)$ and $1(2 \%)$ certolizumab.

Of the 55 confirmed infections: non-pneumonic infection in $13(24 \%)$ patients, pneumonia: 10 (18\%), septic arthritis: $6(11 \%)$, septic shock and/or bacteremia: 6 (11\%), abscess: $4(7 \%)$, urinary infections: $4(7 \%)$, cellulitis: $3(5 \%)$, cutaneous leishmaniasis: $3(5 \%)$, acute gastroenteritis: $2(4 \%)$, surgical wound infection: 1 (2\%), cutaneous infection: 1 (2\%), Septic bursitis: $1(2 \%)$, gonorrhoea: $1(2 \%)$. The mean time of hospital admission was 9.76 days. Three $(7 \%)$ patients presented the infection within a year of starting treatment.

The rate of severe infection x100 patients/year of exposure is 2.01 (1.47-2.67). The odd ratio of admission for infection of $3.67(1.11-4.87, p=0.03)$. The risk of admission for infection in patients with peripheral arthritis (RA, PSA, JIA) is 2.42 (1.21-3.11) times higher than in patients with $A S(p=0.012)$.

In the table 1, the Odd ratio of income for infection distributed by anti-TNF drug is shown.

\begin{tabular}{lcc}
\hline Anti-TNF & $\begin{array}{c}\mathbf{N}^{\circ} \\
\text { patients }\end{array}$ & $\begin{array}{c}\text { Odds ratio (IC } \\
\text { 95\%) }\end{array}$ \\
\hline Infliximab & 101 & $8.01(4.99-12.05)$ \\
Golimumab & 82 & $5.68(2.95-9.73)$ \\
Certolizumab & 33 & $3.25(0.54-10.03)$ \\
Adalimumab & 239 & $3.03(2.08-4.22)$ \\
Etanercept & 188 & $2.44(1.48-3.76)$ \\
\hline
\end{tabular}

Conclusions: 1 . The severe infection rate $\times 100$ patients/year of exposure is 2.01 and the prevalence is $9.6 \%$. 2. The majority of severe infection occurred late, after more than 1 year of treatment. 3. The most frequent infection were those of respiratory origin, followed by sepsis or bacteremia and septic arthritis. 4. Etarnecept has presented the lowest rate of severe infection. 5. Patients with AS have a lower risk of severe infection than patients with chronic peripheral arthritis.

Acknowledgements: The study was supported with a research grant from the Association for Research in Rheumatology of Marina Baixa (AIRE-MB).

Disclosure of Interest: None declared

DOI: 10.1136/annrheumdis-2018-eular.4971

\section{SAT0402 \\ LEISHMANIASIS IN PATIENTS ON TUMOUR NECROSIS FACTOR INHIBITORS TREATMENT}

L. Montolio Chiva ${ }^{1}$, E. Valls Pascual ${ }^{1}$, D. Ybañez Garcia ${ }^{1}$, A. Martínez Ferrer ${ }^{1}$, M. Fernández Matilla ${ }^{2}$, J. Lluch Pons ${ }^{3}$, X. Juanola Roura ${ }^{3}$, J. Marcoval Caus ${ }^{4}$, M.-I. García Briz ${ }^{5}$, J.M. Paredes Arquiola ${ }^{6}$, I. López Cruz ${ }^{7}$, A. Salazar Cifre ${ }^{8}$, M. Aguilar Zamora ${ }^{1}$, A.V. Orenes Vera ${ }^{1}$, A. Garcia Sendra ${ }^{1}$, V. Núñez Monje', I. Torner Hernández ${ }^{1}$, J.J. Alegre Sancho ${ }^{1} .{ }^{1}$ Rheumatology, Hospital Dr Peset, ${ }^{2}$ Rheumatology, Hospital Arnau de Vilanova, Valencia; ${ }^{3}$ Rheumatology;

${ }^{4}$ Dermatology, Hospital de Bellvitge, Barcelona; ${ }^{5}$ Dermatology; ${ }^{6}$ Digestive; ${ }^{7}$ Internal Medicine, Hospital Dr Peset, ${ }^{8}$ Epidemiology, Public Health Center, Valencia, Spain

Background: Tumour necrosis factor (TNF) plays a major role in defense against leishmaniasis. Despite wide use of TNF $\alpha$ inhibitor (anti-TNF) for several diseases, leishmaniasis has been a rare infectious complication so far in these patients. Recently, an increased incidence has been noted.

Objectives: To describe a recent multicenter case series of leishmaniasis in patients with chronic inflammatory diseases treated with anti-TNF.

Methods: We reviewed the clinical history of a multicentric series of patients with chronic inflammatory diseases treated with anti-TNF, who were diagnosed with leishmaniasis between January 2013 and December 2017. Patients came from Rheumatology, Digestive and Dermatology departments of several hospitals in Valencia $^{2}$ and Cataluña ${ }^{1}$ region. Demographic (age, sex) and clinical (inflammatory disease, comorbidities, current treatment, year of infection and leishmaniasis form) variables were collected. Anti-TNF withdraw, subsequent reintroduction and recurrence rate were recorded in two hospitals. Biologic drug dispensation trends from 2013 to 2016 and epidemiological data published by the Regional Ministry of Health of Valencia for the area where cases were most incident were analysed.

Results: 25 cases of leishmaniasis in patients treated with immunomodulators were identified:7 on DMARD, 1 on tocilizumab and 17 on anti-TNF ( 7 infliximab, 4 adalimumab, 3 golimumab, 2 certolizumab, 1 etanercept). Regarding patients on anti-TNF, 2 cases were collected in 2014, 4 in 2015, 4 in 2016 and 7 in 2017 Three patients developed the visceral form, 13 the cutaneous form and 1 presented visceral and cutaneous involvement. Seven patients were males and 10 females, with an average age of 50 (SD14) years. One patient presented rheumatoid arthritis, 4 psoriatic arthritis, 1 undifferentiated spondyloarthropathy, 2 ankylosing spondylitis, 1 uveitis, 6 Crohn's disease and 2 ulcerative colitis. Six patients presented other chronic disease (1 latent tuberculosis, 1 pyoderma gangrenosum, 1 psoriasis and 3 diabetes mellitus). In two hospitals (15 patients), anti-TNF treatment was withdrawn in 10 cases, and it was reintroduced after treating the infection in 5 cases. No infection recurrences have been indentified. Focusing on the area with the highest incidence of cases, despite the increase in anti-TNF use over the last years, its consumption was not parallel to the rise of leishmaniasis cases reported.

Conclusions: the disproportionate increase of leishmaniasis cases in patients with anti-TNF suggests the necessity to investigate and control other possible factors involved.

Disclosure of Interest: None declared

DOI: 10.1136/annrheumdis-2018-eular.6867

\section{SAT0403 BLOOD B CELL SUBSET PROFILE DISTURBANCE IN WHIPPLE'S DISEASE}

M. Le Goff ${ }^{1}$, D. Cornec ${ }^{2}$, D. Guellec ${ }^{1}$, T. Marhadour $^{1}$, V. Devauchelle-Pensec ${ }^{1}$, S. Jousse-Joulin ${ }^{1}$, M. Herbette ${ }^{1}$, J.M. Cauvin ${ }^{3}$, C. Le Guillou ${ }^{3}$, Y. Renaudineau ${ }^{4}$, J.O. Pers ${ }^{4}$, A. Saraux ${ }^{1} .{ }^{1}$ Rheumatology, CHU Brest and Université Bretagne Occidentale; ${ }^{2}$ Rheumatology, CHU Brest and UMR 1227 Université Bretagne Occidentale; ${ }^{3} \mathrm{DIM} ;{ }^{4}$ Immunology, CHU Brest and Université Bretagne Occidentale, Brest, France

Background: Technological advances have improved phenotypical characterisation of blood cells, and flow cytometry is currently used in haematology, infectious disease, systemic auto-immune diseases. Abnormalities of blood B cell subset profile might provide a useful diagnostic tool in systemic auto-immune diseases, especially for primary Sjögren's Syndrome ${ }^{1}$ in which the activated B cells to memory B cells ratio is increased. Nevertheless, we observed that some patients suffering from chronic infection had lymphocytes disturbances similar to those observed in primary Sjögren's Syndrome.

Objectives: Whipple'disease (WD) is a rare, systemic, disease caused by intracellular gram positive bacterium, Tropheryma Whipplei (TW). No previous study evaluated the role of B cells in WD. The aim of this study was to analyse whether the circulating blood B cell subset disturbances is characteristic of WD. 
Methods: We collected characteristics of all patients coming for inflammatory rheumatism in our rheumatology department between April 2010 and December 2016. All of them had systematically routine examination, immunological tests, lymphocyte subsets in peripheral blood by flow cytometric analysis. We selected among this population those patients who also had PCR for TW for suspicion of WD, and compared the distribution of lymphocyte subsets of those with and without WD. Then, we evaluated their diagnostic value for WD using a ROC curve. Results: Among 3494 patients with inflammatory rheumatism, 121 patients (212 visits) had a suspicion of WD and the diagnosis of WD was retained by an expert rheumatologist for 9 (7.4\%) (22 visits). T cells and NK cells were not different whereas percentage of circulating memory $B$ cells (lgD $\left.{ }^{-} C^{2} 38 l o w\right)$ was lower $(18.0 \% \pm 9.7 \%$ vs $26.0 \pm 14.2 \%, p=0.041)$ and the ratio of activated $B$ cells to memory $B$ cells higher $(4.4 \pm 2.0$ vs $2.9 \pm 2.2, p=0.023)$, in patients compared with controls. More precisely, the analysis of the frequency of peripheral blood $B$ cells showed that $\operatorname{lgD}+\mathrm{CD} 27$ - naïve B cells were higher $(66.2 \% \pm 18.2 \%$ vs 54.6 $\pm 18.4 \%, p=0.047)$ and $\operatorname{lgD}-\mathrm{CD} 27$ +switched memory $\mathrm{B}$ cells lower $(13.3 \%$ $\pm 5.7 \%$ vs $21.4 \pm 11.9 \%, p=0.023$ ), in patients compared with the controls. The best diagnostic value was obtained for the lgD +CD27- naïve B cells (cut off 70.5, sensitivity $73 \%$, specificity $80 \%$ ).

Conclusions: Our study provides data on blood B cells disturbances and a first step towards understandings of immunological abnormalities in WD. These disturbances provide guidance for diagnosis and allow physiopathological hypothesis.

\section{REFERENCE:}

[1] Binard A, Le Pottier L, Devauchelle-Pensec V, Saraux A, Youinou P, Pers $\mathrm{J}-\mathrm{O}$. Is the blood B-cell subset profile diagnostic for Sjogren syndrome? Ann Rheum Dis 2009;68(9):1447-52.

Disclosure of Interest: None declared DOI: 10.1136/annrheumdis-2018-eular.3191

\section{SAT0404 CLINICAL CHARACTERISTICS AND OUTCOME AFTER TREATMENT OF A NATIONAL COHORT OF PCR- POSITIVE LYME ARTHRITIS}

M. Scherlinger ${ }^{1,2}$, A. Grillon ${ }^{3}$, J. Sibilia ${ }^{4}$, L. Arnaud ${ }^{4}$, B. Jaulhac ${ }^{3} .{ }^{1}$ Rheumatology, CHU de Bordeaux; ${ }^{2}$ Immunoconcept, UMR-CNRS 5164, Bordeaux; ${ }^{3}$ Centre National de Référence Borrelia; ${ }^{4}$ Rheumatology, CHU de Strasbourg, Strasbourg, France

Background: Lyme arthritis (LA) is a disseminated Borrelia infection whom prevalence is lower in Europe than in the USA, probably because of difference in Borrelia species ecology. Few data concerning treatment efficacy and long-term outcome of LA in Europe are available.

Objectives: The aim of our study was to describe clinical characteristics and treatment outcomes of a national cohort of patients with LA confirmed with synovial fluid PCR.

Methods: We conducted a retrospective observational study using the French Borrelia reference centre database. Patients presenting with a PCR positive for Borrelia DNA in their synovial fluid between 2011 and 2016 were included. PCRpositive patients were offered by their referring physician to participate to a standardised telephonic interview. Patients' medical files were also retrieved. The main objectives were to describe patient characteristics, disease presentation and outcomes after antibiotic treatment.

Results: Between 2011 and 2016, among 358 synovial fluids tested at the national reference centre, 38 were positive for Borrelia DNA. Among these patients, 35 were contacted ( 3 missing contact information). Median age was 36 years with $31 \%$ minors and $63 \%$ men. Tick exposure was reported by $88 \%$ patients whereas tick bite and erythema migrans were only reported in $40 \%$ (10/ 25 ) and $14 \%(3 / 21)$, respectively. The presentation was monoarticular in $91 \%$ (32/ $35)$ cases and oligoarticular in others. The knee was involved in $97 \%(34 / 35)$ cases and $21 \%$ presented fever. The diagnosis was often delayed with a median time from symtom onset to diagnosis of 3 months (range 1 to 112). The serology performed before or at the time of the PCR testing was lgG positive in all cases but only in IgM positive in $40 \%$. All positive IgG serologies were also positive with Western-Blot. In the synovial fluid, the identified species of Borrelia were $B$. burgdorferi sensu stricto, B. garinii and B. afzelii in $54 \%, 29 \%$ et $17 \%$ of cases, respectively. Antibiotics prescribed were mostly doxycycline and ceftriaxone in 17 and 9 patients, respectively, sometime in combination. Follow-up data were available for 26 patients with a median follow-up time of 27 months (range 1-73). Full recovery was reported by $62 \%$ patients whereas 6 presented persistent non-inflammatory articular pain of the affected joint. One patient reported a chronic pain syndrome without objective sign of persistent infection (including a negative synovial fluid PCR). Three patients developed chronic inflammatory arthritis leading to the introduction of DMARDs.
Conclusions: Our study reports original data on Lyme arthritis in France. Treatment outcomes are usually good but a significant proportion of patients may develop chronic inflammatory arthritis.

Acknowledgements: All clinicians participating the study.

Disclosure of Interest: None declared

DOI: 10.1136/annrheumdis-2018-eular.2006

\section{SAT0405 1 FINDINGS OF A COHORT OF PATIENTS WITH CHIKUNGUNYA IN A COLOMBIAN POPULATION}

M.A. López ${ }^{1}$, R. Badillo ${ }^{1,2}$ on behalf of Association Colombian of Rheumatology, E. Rojas ${ }^{1}$, M. Gelvez ${ }^{1}$, Y. Santamaría ${ }^{1}$ on behalf of Association Colombian of rheumatology. ${ }^{1}$ Santander, Industrial University of Santander, ${ }^{2}$ Santander, Association Colombian of Rheumatology, Bucaramanga, Colombia

Background: Chikungunya virus is a Togaviridae family virus transmitted by mosquitoes, which generates febrile syndrome with joint pain. It has been widely studied for the findings of chronic inflammatory polyarthropathy similar to rheumatoid arthritis. In Colombia, an epidemic occurred between 2014 and 2015, which was studied in several cities. International meta-analyses have shown a prevalence of $32.13 \%$ in the follow-up cohorts greater than 18 months. At present, this issue has gained a new opportunity due to the appearance of new outbreaks in Italy and France, after 10 years of the first epidemic.

Objectives: To compare the clinical findings of a cohort of patients with Chikun gunya in the subacute phase and the chronic phase.

Methods: Follow-up of 70 patients who attended Chikungunya in a Colombian population who were evaluated in person by a rheumatologist, initially at 40 days after the disease and after two years.

Results: The average age of the study participants was 59.88 years, being more frequent in women with $78.6 \%$ of the cases. $40 \%$ of the cases were older than 65 years, with the older adult population being a representative part of the cases.

The history of osteoarthrosis occurred in $11.7 \%$ of cases. There was no history of systemic lupus erythematosus.

The most frequent symptoms presented at the first visit (outbreak context) were as follows, in order: Joint pain $(71.4 \%)$, morning stiffness (48.6\%), Metacarpophalangeal compression test $(51.2 \%)$. The most frequent symptoms in the second visit (two years after the outbreak) were: joint pain $(74.2 \%)$, morning stiffness (21.4\%) and metacarpophalangeal compression test (17.1\%)

At the time of the second visit, the clinical findings were classified by diseases, according to the rheumatologist's assessment as follows: Post-Chikungunya polyarthropathy (17.1\%), Fibromyalgia (10\%), Carpal tunnel syndrome $(17.1 \%)$ Osteoarthritis of knees (32.8), osteoarthritis of distal interphalangeal (20\%), painful shoulder syndrome $(17.1 \%)$, tenosynovitis $(18.6 \%)$, gout $(1.4 \%)$, sequelae of fracture of hip (1.4\%), lateral epicondylitis (1.4\%). $28.5 \%$ of the cases had no diag nosis of rheumatological pathology. Of the total cases, only $24.3 \%$ (17 people) had symptoms for more than 6 weeks.

Conclusions: Chikungunya virus infection increases the prevalence of joint and extra-articular rheumatological diseases in the Colombian population evaluated.

\section{REFERENCES :}

[1] Rodríguez- Morales A, et al. Prevalence of post-Chikungunya Chronic Inflammatory Rheumatism: A Systematic Review and Meta-Analysis 2016.

[2] Campion E. Chikungunya Virus and the Global Spread of a MosquitoBorne Disease. NEJM 2015.

[3] CDC. Chikungunya in Italy 2017.

Acknowledgements: Association Colombian of rheumatology The epidemiological research centre, Industrial University of Santander. Disclosure of Interest: None declared DOI: 10.1136/annrheumdis-2018-eular.7482

\section{SAT0406 CHIKUNGUNYA VIRUS AND THE RHEUMATOLOGY: OBSERVATION OF 76 CASES DURING AN EPIDEMIC IN BRAZIL}

M. C. D. F.Salgado, Y. D. F. B.Chagas, J.L.P. Vaz, F.C. Freire, V. Vigneron, A. Vaz. General Medicine, Federal University of Rio de Janeiro State, Rio De Janeiro, Brazil

Background: Chikungunya fever is caused by a virus of the family Togaviridae and the genus Alphavirus, The first epidemic occurred in Africa in 1952, transmitted by mosquitoes of the genus Aedes. In Brazil, the first cases were registered in 2014. Clinical manifestations include fever, polyarthralgia, joint oedema, arthritis and morning stiffness.

Diagnosis is confirmed by $\operatorname{lgM} / \mathrm{lgG}$ serology for Chikungunya. The persistence of joint symptoms for a long time is an important feature of the disease. 